\title{
Primordial gravitational wave and DECIGO
}

\author{
Seiji Kawamura ${ }^{1}$ \\ Nagoya University \\ Furo-cho, Chikusa-ku, Nagoya, Aichi, Japan \\ E-mail: kawamura@u.phys.nagoya-u.ac.jp
}

\section{DECIGO working group}

Gravitational waves were detected in 2015 for the first time in the history of humankind. Since then, around ten gravitational wave signals coming from black hole/neutron star binary coalescences have been detected. Among various expected gravitational wave sources, the beginning of the universe is one of the most exciting targets. The inflation theory was developed to solve various problems of the big bang model such as the horizon problem. According to the inflation theory, the primordial gravitational waves produced during the inflation period could reach us. Deci-hertz Interferometer Gravitational Wave Observatory (DECIGO), is the future Japanese mission, which aims at detecting the primordial gravitation waves between $0.1 \mathrm{~Hz}$ and $10 \mathrm{~Hz}$ as well as obtaining other important sciences. DECIGO has a 1,000-km arm length and Fabry-Perot cavities implemented to improve the sensitivity. DECIGO employs drag-free spacecraft in a heliocentric earth-trail orbit. We plan to launch B-DECIGO first to demonstrate technologies necessary for DECIGO, as well as to detect a variety of gravitational waves. Then we plan to launch DECIGO to detect the primordial gravitational waves.

The 4th KMI International Symposium (KMI2019)

18-20, February 2019

Nagoya, Japan

\section{${ }^{1}$ Speaker}




\section{Introduction}

The existence of gravitational wave was predicted by A. Einstein in 1916. According to the general theory of relativity, gravitational waves propagate as a tidal distortion of spacetime at the speed of light, and thus gravitational waves change a distance between two masses. The theory also predicts that gravitational waves are emitted from accelerating bodies, such as neutron star binary coalescences, black hole binary coalescences, supernovae, and the inflation of the universe.

For the detection of gravitational waves, a Michelson laser interferometer with suspended mirrors is often used. The detection mechanism is very simple; gravitational waves shake the mirrors by a tiny amount, and thus the interfering light slightly changes its brightness, which is measured by a photodetector. One of the outstanding merits of using the Michelson laser interferometer comes from the fact that the gravitational wave signals can be made larger just by extending the arm length of the interferometer. However, because the longest arm length possible to realize on the earth is still too short for gravitational waves with an interesting frequency range, a Fabry-Perot cavity is implemented to extend the effective arm length further.

Currently, several large-scale laser interferometric gravitational wave detectors have been and are being constructed around the world: LIGO (in the US), Virgo (in Italy), GEO (in Germany), KAGRA (in Japan), and Indigo (in India). Among them, LIGO is currently the most advanced one. The arm length of LIGO is $4 \mathrm{~km}$, and two detectors are located in Washington state and Louisiana state.

LIGO succeeded in detecting gravitational waves for the first time in the history of humankind in 2015 [1]. According to the detailed analysis, it was found that the detected gravitational waves were emitted from the black hole binary coalescence at $1.3 \mathrm{G}$ light-year distance from the earth. It was also found that the mass of the two black holes was 29 and 36 solar mass, respectively, and the mass of the large black hole after the coalescence was 62 solar mass. This indicates that energy corresponding to 3 solar mass was emitted as gravitational waves. Because of this detection, Rai Weiss, Kip Thorne, and Barry Barish were awarded Nobel prize in 2017.

In 2017, LIGO together with Virgo also detected gravitational waves coming from the neutron star binary coalescence [2]. This time 1.7 second later after the detection of the gravitational wave, a short gamma-ray burst was also detected in the same direction as the gravitational wave signal [3]. Ten hours later a new astronomical object was found by the electromagnetic wave observation in the same direction [4]. The follow-up observations by around 70 telescopes were found to be consistent with the hypothesis that the short gamma-ray burst is emitted from a neutron star binary coalescence and the so-called r-process takes place in the system. This event was the beginning of the multi-messenger astronomy.

Since the first detection of gravitational waves, LIGO, partially together with Virgo, detected around ten gravitational wave signals. Most of them were from the black hole binary coalescences except the only one from the neutron star binary coalescence. It is expected that LIGO, Virgo, and KAGRA shortly will make more frequent detection with a better signal-to-noise ratio, which could lead us to more fruitful multi-messenger astronomy.

Unfortunately, the primordial gravitational waves coming from the inflation period are not considered as a promising gravitational wave target for those terrestrial detectors, because the sensitivity of the terrestrial detectors is not good enough for the primordial gravitational waves. 
Therefore, the idea of space gravitational wave antenna, DECIGO, was brought to detect the primordial gravitational waves. In this paper, the inflation and the primordial gravitational waves are explained in section 2, and the aimed science and design of DECIGO are explained in section 3.

\section{Inflation and primordial gravitational wave}

The inflation theory was developed to solve various problems of the big bang model such as the horizon problem, flatness problem, and monopole problem [5] [6]. The horizon problem, for example, is that the incredible homogeneity of the universe at $380 \mathrm{k}$ year from the beginning of the universe observed with cosmic microwave background by Planck satellite, etc. cannot be explained because the causality range at that time was much smaller. If the expansion of the universe at the very early stage of the universe was exponentially accelerated, however, the causality range at $380 \mathrm{k}$ year could be much larger, and the observed homogeneity can be explained.

During the inflation period, it is reasonable to assume that the quantum fluctuations of the space-time can produce the quantum gravitational waves. Such gravitational waves are supposed to appear at a certain moment and disappear at the next moment because of their quantum nature. However, if a gravitational wave, whose wavelength was slightly smaller than the horizon size, was produced at a certain moment, the wavelength of the gravitational wave becomes larger than the horizon size at the next moment, because of the exponential expansion of the universe during the inflation period. Such gravitational wave could change its nature from quantum to classical and could have its amplitude frozen to be constant. After the inflation, the universe expands much more slowly compared with the inflation period, and when the wavelength of the gravitational wave becomes smaller than the horizon range, the gravitational wave starts propagating and eventually reaches us. It should be noted that the gravitational wave produced at the early stage of the inflation period has a longer wavelength, thus lower frequency, and it starts propagating more recently, while the gravitational wave produced at the later stage of the inflation period has a shorter wavelength, thus higher frequency, and it starts propagating more formerly.

The spectrum of the primordial gravitational wave is determined by the details of the inflation as well as the expansion of the universe after the inflation. Therefore, if we detect primordial gravitational waves, we could reveal the details of the birth of the universe, such as whether or not the inflation occurred, which model of the inflation is correct, and when the inflation ended.

\section{Space gravitational wave antenna DECIGO}

\subsection{Gravitational wave detector in space}

There are strong advantages of employing a gravitational wave detector in space. First, much longer arm length can be realized in space compared with the ground-based detector. On the earth, the arm length is limited below several tens km because of the curvature of the earth. Besides, the vacuum system covering the whole system could be extremely costly. In space, however, it is possible to extend the arm length significantly longer, because there is no land limit and no vacuum system is necessary for space. Because the gravitational wave signal in an interferometer is proportional to the arm length of the interferometer, the gravitational wave signal can be increased in space. It should be noted, however, that this increase of the signal is only effective at lower frequencies because at higher frequencies the gravitational wave changes its phase while 
the laser light is still propagating in the interferometer, which causes partial cancellation of the signal in the interferometer.

Secondly, the noise at lower frequencies is much smaller in space than the ground-based detectors. On the earth, the mirrors are shaken by the ground motion and the suspension thermal noise, because the mirrors are suspended by wire. In space the mirrors can float in spacecraft; thus they do not have to be suspended. Therefore, the seismic noise and the suspension thermal noise do not exist in space. Because those noise sources limit the sensitivity of the ground-based detectors at lower frequencies, the space detectors have much lower noise at lower frequencies.

Because of the two reasons mentioned above, the space detectors have a much better signalto-noise ratio at lower frequencies compared with the ground-based detectors. Moreover, it should also be noted that in principle we can expect larger gravitational wave signals at lower frequencies, because heavier objects, which move more slowly in general, emit larger gravitational waves.

\subsection{LISA}

Laser Interferometer Space Antenna (LISA) is a future mission in space [7]. Among various science targets of LISA, one of the most important objectives is to detect gravitational waves coming from intermediate-mass to giant black hole binaries to reveal the formation mechanism of giant black holes in the center of galaxies. LISA has three spacecraft, which contains mirrors. A change in the distance of the mirrors caused by gravitational waves is measured by an interferometer. The arm length of LISA is 2.5 million $\mathrm{km}$, and because of the refraction of the laser light, $1 \mathrm{~W}$ of the laser power is sent from one satellite and only about $10 \mathrm{pW}$ of the power reaches another satellite. Therefore, the light transponder technique is used; an additional laser is phase-locked to the incoming beam as if the beam is reflected by a mirror. The LISA Pathfinder was already launched to verify the technologies required for LISA. It successfully demonstrated that the acceleration noise satisfied the requirement for LISA [8].

\subsection{DECIGO}

Deci-hertz Interferometer Gravitational Wave Observatory (DECIGO) is the future Japanese mission in space [9] [10]. The observation frequency band is 0.1 to $10 \mathrm{~Hz}$, which is just between the LISA band and the groundbased detector band. Therefore, DECIGO can play a follow-up role for gravitational waves coming from compact binaries observed by LISA, as well as can play a prediction role for such gravitational waves to be observed by ground-based detectors. Moreover, the outstanding advantage of this frequency band is that there is no confusion limiting noise caused by too many gravitational wave signals coming

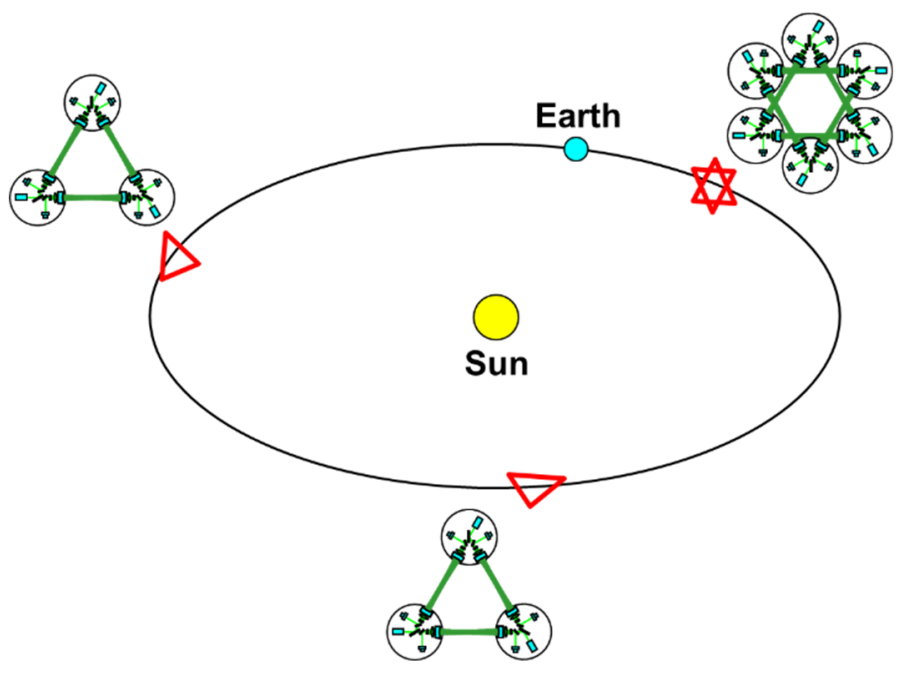

Fig. 1. Orbit of DECIGO. 
from white dwarf binaries, etc. in our galaxy, because white dwarf binaries cannot emit gravitational waves above $0.1 \mathrm{~Hz}$.

DECIGO consists of four clusters of spacecraft, each of which consists of three spacecraft with three interferometers. Two clusters are placed at the same place in a heliocentric earth-trail orbit, while the other two are placed at different places in the same orbit (See Fig. 1). Each spacecraft contains mirrors floating inside the spacecraft, and a change in the distance between mirrors caused by gravitational waves is measured by an interferometer. The interferometer is a

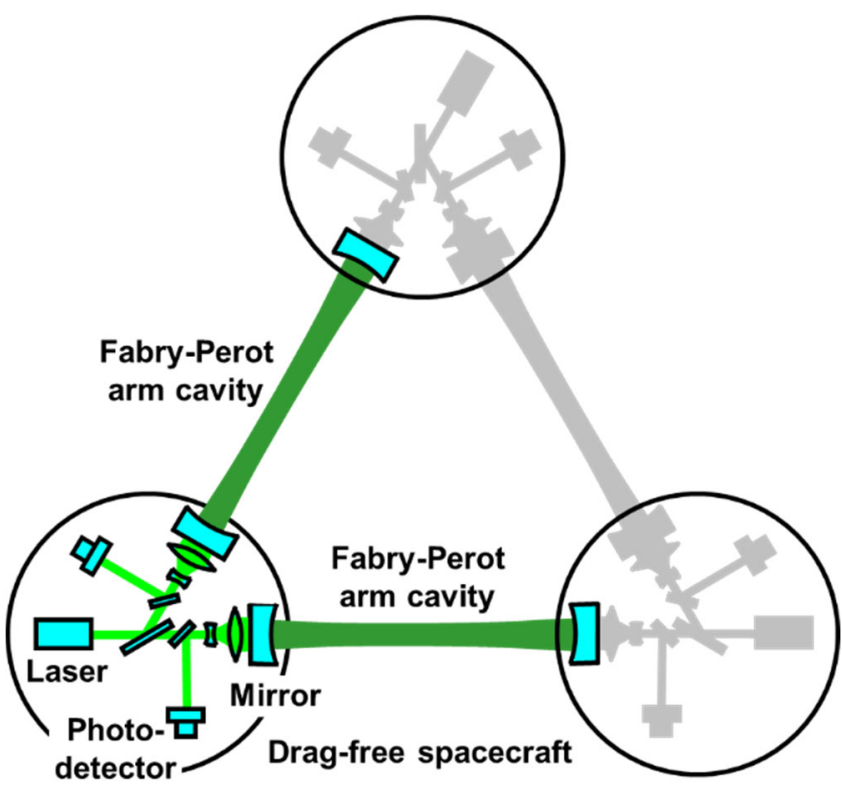

Fig. 2. Pre-conceptual design of one cluster of DECIGO.

Michelson interferometer with a Fabry-Perot cavity to increase the sensitivity of the detector (See Fig. 2). The arm length of DECIGO is $1,000 \mathrm{~km}$, the mirror diameter is $1 \mathrm{~m}$, the mirror mass is $100 \mathrm{~kg}$, the laser wavelength is $0.515 \mu \mathrm{m}$, the laser power is $10 \mathrm{~W}$, and the finesse of the FabryPerot cavity is 10. Each spacecraft has a drag-free system; the mirrors inside the spacecraft are protected from the solar radiation and drag by the outer spacecraft.

The reason why DECIGO adopted an arm length significantly smaller than LISA and a Fabry-Perot cavity instead of the light transponder system is the following (See Fig. 3). In any advanced gravitational wave detectors, the most important noise source is the quantum noise. The

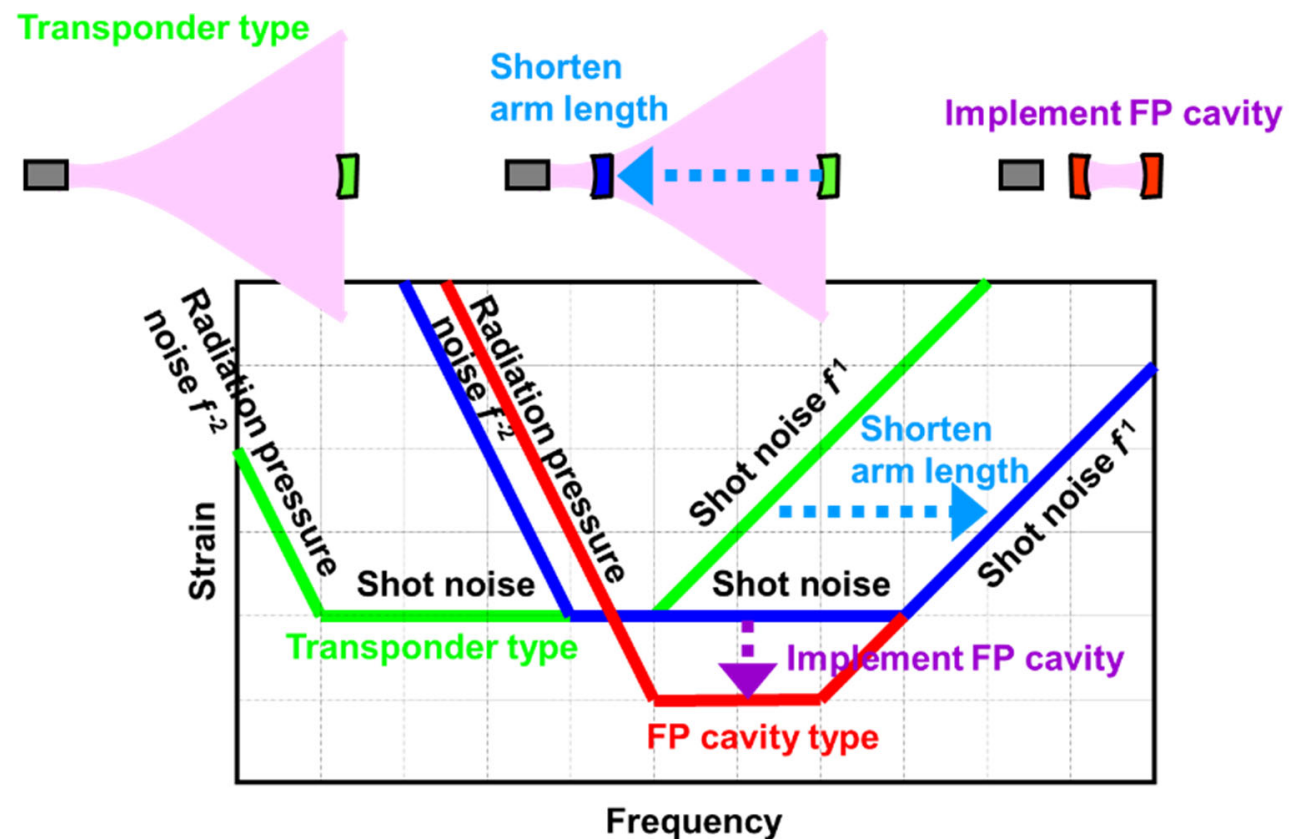

Fig. 3. Quantum noise for the longer arm-length light-transponder type interferometer, the shorter arm-length interferometer, the Fabry-Perot cavity type interferometer. 
quantum noise consists of shot noise and radiation pressure noise. Therefore, it is important to design a detector so that the quantum noise limited sensitivity is optimized. First, let us consider the arm length dependence of the shot noise in terms of strain. If the arm length is relatively long, only a fraction of the laser power reaches another spacecraft. In such a condition, the strain shot noise does not depend on the arm length, because the two opposite effects, the refraction power loss and the gravitational wave signal increase by longer arm length, cancel each other. The corner frequency for the strain shot noise, above which the strain shot noise is worse because of the gravitational wave signal cancellation, is inversely proportional to the arm length. The strain radiation pressure noise is larger with shorter arm length because of the higher laser power and the weaker gravitational wave signals. As a result, the cross-over frequency between the strain shot noise and the strain radiation pressure noise is inversely proportional to the arm length. Therefore, the quantum-noise-limited strain sensitivity curve shifts to higher frequencies with a shorter arm length, maintaining its shape on a logarithmic scale. Moreover, if the arm length is short enough so that all the emitted light power reaches a mirror in another spacecraft, the strain shot noise can be further improved below the corner frequency by implementing a Fabry-Perot cavity at the expense of the strain radiation pressure noise. This is why a shorter arm length with a Fabry-Perot cavity was adopted for DECIGO.

It is also important to explain the drag-free system and the Fabry-Perot cavity locking system are compatible (See Fig. 4). The relative position of the spacecraft with respect to the mirror is measured by a local sensor, and this signal, containing the solar radiation noise and drag noise, is fed back to the thruster of the spacecraft so that the spacecraft follows the motion of the mirror.

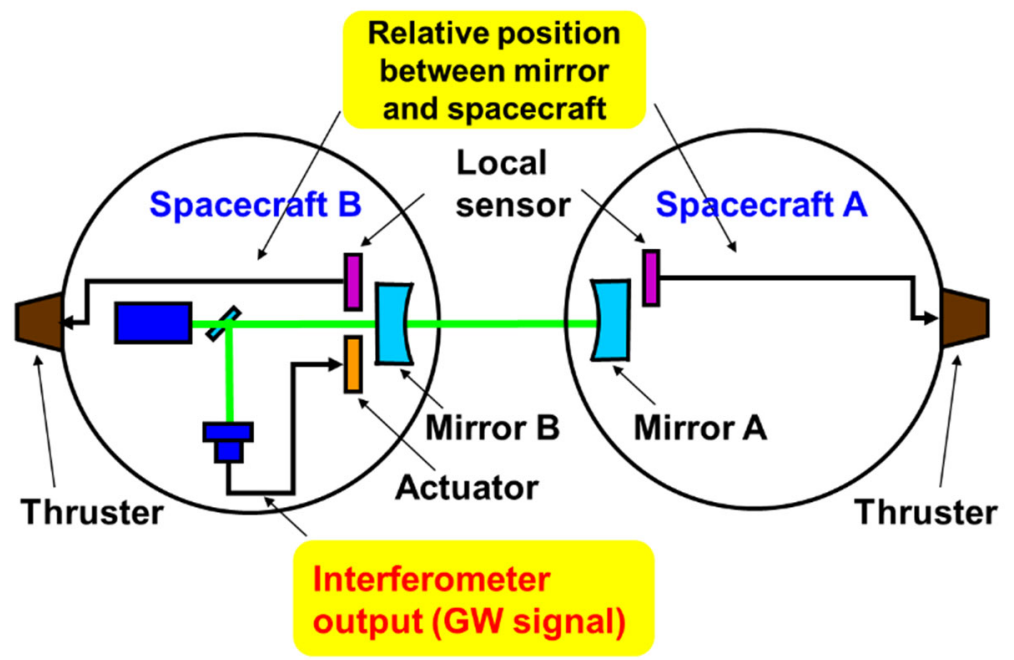

Fig. 4. Drag-free system and Fabry-Perot cavity control system.

The relative position between the two mirrors is measured by the interferometer, and this signal, containing the gravitational wave signal, is fed back to one of the mirrors. The important point is that this signal, containing the gravitational wave signal, does not have the solar radiation pressure noise and the dragging noise.

The target strain sensitivity of DECIGO is $4 \times 10^{-24} \mathrm{~Hz}^{-1 / 2}$ between $0.1 \mathrm{~Hz}$ and $10 \mathrm{~Hz}$ for one cluster, and $1 \times 10^{-25} \mathrm{~Hz}^{-1 / 2}$ between $0.1 \mathrm{~Hz}$ and $10 \mathrm{~Hz}$ with a three-year correlation (See Fig. 5). To accomplish this sensitivity, the force noise requirement for DECIGO is 25 times more stringent than LISA. This number comes from the fact that the strain force noise of DECIGO is comparable with LISA, the arm length of DECIGO is 2,500 times shorter than LISA, and the mirror mass of DECIGO is 100 times heavier than LISA. As for the sensor noise, DECIGO has 30 times looser requirement than ground-based detectors such as KAGRA. This number comes from the fact that 
the strain sensor noise of DECIGO is comparable with KAGRA and, the storage time of DECIGO is 30 times longer than KAGRA.

Some of the science targets expected by DECIGO is the following. (1) The acceleration of the expansion of the universe can be measured directly by detecting gravitational waves coming from neutron star binaries at $\mathrm{z}$ of 1 . This could lead to a better understanding of dark energy. (2) The general relativity can be examined with an accuracy of $10^{4}$ better than before by measuring gravitational waves coming from

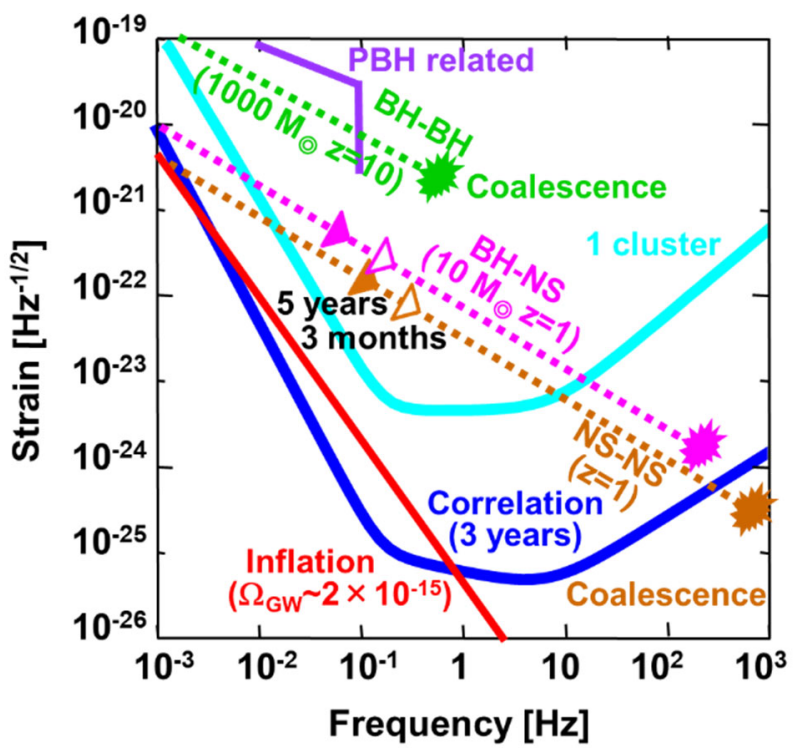

Fig. 5. Target sensitivity of DECIGO and expected gravitational wave signals.

black hole (10 solar mass) neutron star binary at $\mathrm{z}$ of 1. (3) If the primordial black holes can explain some of the dark matters, the fluctuations of the mass distribution, which produced the primordial black holes, were supposed to emit gravitational waves. If DECIGO detects such gravitational waves, it could reveal the secret of the dark matters.

Among the various science targets, the most important objective of DECIGO is to detect the primordial gravitational waves coming from the inflation period. If we succeed in the detection, we could tell whether or not the inflation happened, and if it happened, which inflation model is correct [11], when the inflation ended [12]. We could also obtain the knowledge about the parity violation because DECIGO can tell the difference between the clockwise and counterclockwise gravitational waves [13]. We could also separate the tensor, vector, and scalar mode of gravitational waves [14].

It should be noted here that the expected upper limit of the primordial gravitational wave with the standard inflation model has been decreased after the observation by the Planck satellite. Therefore, the target sensitivity of DECIGO should be improved to ensure a certain detection of the primordial gravitational waves. For that purpose, we are now updating the optical and mechanical design of DECIGO even with the help of the squeezing technologies.

We plan to launch B-DECIGO first to demonstrate technologies necessary for DECIGO, such as a drag-free control system, formation flight, Fabry-Perot cavity in space, etc., as well as to detect a variety of gravitational waves [15]. B-DECIGO has one cluster, which consists of three spacecraft with three interferometers. The orbit of B-DECIGO is now under discussion. The target sensitivity of B-DECIGO is a factor of ten worse than DECIGO. Although B-DECIGO is not designed to detect the primordial gravitational waves, it could put a significant upper limit. After B-DECIGO, we plan to launch DECIGO to detect the primordial gravitational waves.

\section{Conclusions}

The primordial gravitational waves coming from the inflation period carry important information about the beginning of the universe. DECIGO has been designed to accomplish direct 
observation of the primordial gravitational waves, among a variety of science targets, to reveal the secret of the birth of the Universe. DECIGO is planned to be launched after B-DECIGO, which verifies the technologies necessary for DECIGO.

\section{References}

[1] LIGO Scientific Collab. and Virgo Collab. (B. P. Abbott et al.), Observation of Gravitational Waves from a Binary Black Hole Merger, Phys. Rev. Lett. 116 (2016) 061102

[2] LIGO Scientific Collab. and Virgo Collab. (B. P. Abbott et al.), GW170817: Observation of Gravitational Waves from a Binary Neutron Star Inspiral, Phys. Rev. Lett. 119 (2017) 161101

[3] A. Goldstein et al., An Ordinary Short Gamma-Ray Burst with Extraordinary Implications: FermiGBM Detection of GRB 170817, Astrophys. J. 848 (2017) L14

[4] D. A. Coulter et al., Swope Supernova Survey 2017 a (SSS17a), the optical counterpart to a gravitational wave source, Science 358 (2017) 1556

[5] K. Sato, First-order phase transition of a vacuum and the expansion of the Universe, Mon. Not. R. astr. Soc. 195 (1981) 467

[6] A. H. Guth, Inflationary universe: A possible solution to the horizon and flatness problems, Phys. Rev. D 23 (1981) 347

[7] K. Danzmann et al., LISA and its pathfinder, Nat. Phys. 11 (2015) 613

[8] M. Armano et al., Beyond the Required LISA Free-Fall Performance: New LISA Pathfinder Results down to $20 \mu \mathrm{Hz}$, Phys. Rev. Lett. 120 (2018) 061101

[9] N. Seto, S. Kawamura, and T. Nakamura, Possibility of Direct Measurement of the Acceleration of the Universe Using $0.1 \mathrm{~Hz}$ Band Laser Interferometer Gravitational Wave Antenna in Space, Phys. Rev. Lett, 87 (2001) 221103

[10] S. Kawamura, et al., Space gravitational-wave antennas DECIGO and B-DECIGO, Int. J. Mod. Phys. D 27 (2018) 1845001

[11] S. Kuroyanagi, T. Chiba and N. Sugiyama, Precision calculations of the gravitational wave background spectrum from inflation, Phys. Rev. D 79 (2009) 103501

[12] S. Kuroyanagi, K. Nakayama and J. Yokoyama, Prospects of determination of reheating temperature after inflation by DECIGO, Prog. Theor. Exp. Phys. 2015 (2015) $013 \mathrm{E} 02$

[13] N. Seto, Quest for circular polarization of a gravitational wave background and orbits of laser interferometers in space, Phys. Rev. D 75 (2007) 061302(R)

[14] A. Nishizawa, A. Taruya and S. Kawamura, Cosmological test of gravity with polarizations of stochastic gravitational waves around 0.1-1 Hz, Phys. Rev. D 81 (2010) 104043

[15] T. Nakamura et al., Pre-DECIGO can get the smoking gun to decide the astrophysical or cosmological origin of GW150914-like binary black holes, Prog. Theor. Exp. Phys. 2016 (2016) 093E01 